\title{
LA ESTRUCTURA EN LA TEORÍA VERBOTONAL Y SUPERACIÓN DE ESTRUCTURAS PERCEPTIVAS Y LINGÜÍSTICAS DURANTE EL PROCESO DE LA REHABILITACIÓN
}

Las leyes de la estructura tienen un papel importante ya en el nivel del "funcionamiento" mismo del lenguaje, es decir, en la realización concreta de cada comunicación. Valdría entonces la pena aclarar lo que son "estructuras de funcionamiento". La comprensión "estructural" y la comprensión "funcional" coinciden. ¿Qué significa "comprensión estructural" del lenguaje? La noción de estructura corresponde aquí a la significación habitual del termino psicológico. Se trata de un conjunto formado por fenómenos solidarios. Una estructura puede estar compuesta de elementos muy reducidos y, algunas veces, aun idénticos desde el punto de vista analítico. Lo que realmente cambia los efectos de la estructura es una distinta disposición de los elementos, otra organización.

Las estructuras del lenguaje que mencionamos aquí difieren de aquéllas que se apoyan en los análisis comunmente llamados análisis estructurales del lenguaje. Por eso podemos decir que el método verbotonal no se basa en ninguna de las teorías estructurales del lenguaje ni en la aplicación de alguna de ellas. De aquí resulta la oposición entre la noción psicológica de estructura y el análisis linguiístico de la estructura. En la lingüística contemporánea, la noción de estructura es por una parte, siempre fiel al método analítico de los positivistas y por eso se queda en oposición a otros métodos que comprenden la noción de la estructura en base a otras disciplinas científicas.

El funcionamiento mismo, y cada acto de comunicación, constituye ya una estructura donde están juntos elementos casuales y concretos. El acontecimiento no es tan sólo una ocasión simple para que la estructura del lenguaje se manifieste en uno de los comportamientos, sino que éste mismo entra en la estructura como uno de sus elementos constitutivos. Cada comunicación se organiza, se estructura en función de todos sus componentes, aquí y ahora.

Lo que define la estructura de la comunicación, es que ésta es una "estructura del sentido" (Estructura de signos lingüísticos). Comunicar significa transmitir una significación (un mensaje conteniendo una información). La función esencial del lenguaje es la expresión de la significación por medio del sonido (transmisión del mensaje por medio del sonido). El sonido, dentro del lenguaje, no tiene ningún papel por sí mismo, sino que tan sólo tiene un "papel social": sirve para "vehicular un sentido" (un men- 
saje). La cadena de la comunicación se establece, se organiza para transmitir una significación (información). Las leyes de la estructura de la comunicación son las leyes de la estructura de la transmisión de la significación (transmisión de la información). Lo que se constituye en la comunicación son "unidades de sentido" (unidades de signos linguísticos), o sea las totalidades significantes estructuradas (totalidades de signos estructurados), donde la significación de cada elemento se determina por relación y otros elementos en su totalidad.

Ésta es, en el sentido más amplio de la palabra, la relación que existe entre el sonido y el movimiento por una parte y las significaciones que constituyen el lenguaje, por otra. El sonido y el movimiento o "valores de la lengua hablada", son elementos naturales que el hombre utiliza como portadores de la significación (mensajes conteniendo la información) en el mundo humano: éstos llegaron a ser lenguaje por ser significantes.

La estructura del lenguaje está caracterizada por la ley de búsqueda de lo óptimo: los elementos de la comunicación se estructuran para hacerla lo más eficaz posible. El lenguaje trata de unir de un modo óptimo la inteligibilidad y la riqueza de informaciones por una parte, y la rapidez de la transmisión y limitación de modos necesarios por otra parte. El lenguaje busca la aceleración en la emisión del mensaje así como en su percepción y su integración. (El lenguaje es un “juego combinado por aceleradores de la integración").

El lenguaje está organizado de tal modo que en la recepción de un mensaje no es necesario poner atención en todos los elementos. El esfuerzo de la atención está organizado con momentos de descanso, lo que permite una comprensión más rápida.

Este concepto del lenguaje, comprendido como una estructura organizada en función de lograr lo óptimo, introduce un sentido particular de la estructura. De tal modo, la estructura es definida como una comprensión estructural (funcional) de elementos, como la búsqueda de vías arquitecturales, organizativas; combinaciones que pueden explicar o hacer más eficaz la estructura o el funcionamiento de nuestro cerebro, la estructura de la materia y su utilización por el hombre por medio de estructuras y organizaciones diferentes pero compuestas de elementos reducidos y algunas veces aun idénticos desde el punto de vista analítico.

En la metodología verbotonal, la estructura no tiene el sentido de la presión que la sociedad ejerce sobre nosotros para hacernos aprender un idioma. Tampoco tiene el sentido habitual en la linguística estructural, donde la forma de la lengua se reduce a un conjunto coherente de sus elementos. Al contrario, la estructura está comprendida en el sentido de que nuestra percepción se estructura sobre la base de elementos óptimos de la emisión. Nuestro cerebro no responde al conjunto de estímulos físicos, sino que más bien hace una selección para organizar y unificar las percepciones. Es en este sentido que decimos que el ritmo y la entonación son elementos óptimos para estructurar una buena percepción. Asimismo decimos que para percibir bien un fonema de un idioma extranjero se necesita transmitir al cerebro la banda óptima de este fonema. 
Primero hay que subrayar el hecho de que emitimos y comprendemos el habla globalmente. Es decir que percibimos al mismo tiempo los fonemas, las entonaciones, los ritmos y los gestos. Es preciso añadir que en esta comprensión global, el contexto real tiene un papel muy importante, como también el contexto de civilización y nuestros conocimientos en general. Por consiguiente, llegamos así en nuestra comunicación por el habla a la estructura del conjunto. La estructura del habla se forma en su funcionamiento, en el acto mismo de hablar. Es una especie de estructuración permanente y dinámica. Es en este sentido que se puede comprender el desarrollo histórico del habla y el uso individual de la misma, tanto en la comunicación cotidiana como en la literatura. La estructura del habla, basada en su funcionamiento, organiza sus componentes estructurales en un conjunto no lineal. Al comunicar, nosotros utilizamos las palabras pero también los valores de la lengua hablada (entonación, etc.): por eso, nuestra expresión no es ni lineal, ni horizontal, sino más bien vertical. Al mismo tiempo, y siempre en el mismo eje, utilizamos medios lexicológicos y medios no lexicológicos. Esto es común, ya sea en los enunciados intelectuales o en los afectivos, volviéndose estos últimos expresivos y muy ricos de significado gracias sobre todo a esta no linealidad de la expresión.

La estructura no es una nueva jerarquía, como muchos linguiistas la conciben, sino una nueva "calidad" donde ciertos elementos adquieren un valor que proviene del conjunto de la estructura. Si aplicamos esta idea a una estructura normal del habla, representada por la oración, podremos ver que los sonidos, las formas y las palabras toman valores diferentes en conjuntos estructurados. Así, las palabras puestas en frases y contextos diferentes tienen significados que derivan de la estructura. Los sonidos cambian en sustancia y significado, sobre todo afectivo, en función de estructuras que expresan varias actitudes del sujeto y distintos contextos y situaciones. Finalmente, el ritmo y la entonación con el gesto (el cuerpo) están a la base de toda la estructuración del habla y se realizan globalmente en una frase, donde son percibidos globalmente. Entonces, desde el punto de vista de la comprensión de las estructuras del habla o del valor de su forma, todo es global y todo es estructural en el habla. Todo es estructuro-global.

Es por eso que nosotros concebimos la existencia de la estructura, su aprendizaje y su estudio teórico en el ramo del diálogo. Ordinariamente escuchamos para entender a alguien, pero sobre todo para responderle. Pensamos y hablamos en diálogo. El diálogo no significa necesariamente conversación entre dos personas; existe un estilo de pensamiento y de escritura que representan una especie de diálogo y que puede ser fácilmente traspuesto en diálogo. Todo es diálogo; estamos siempre dialogando. Aunque tengamos la impresión de no conversar con algún ser viviente, siempre respondemos al mundo inanimado. Es un hecho que el lenguaje se presenta como un diálogo continuo. A veces el diálogo puede ser una especie de monólogo interior, como también un diálogo entre el que habla y el mundo que le rodea, entre el hombre y la naturaleza. 
Según Piaget, una estructura es "cuando los elementos están reunidos en una totalidad que presenta ciertas propiedades en cuanto totalidad, y cuando los elementos dependen entera o parcialmente de estos caracteres de la totalidad". Esta definición muestra ya que la totalidad domina los elementos que la componen. Podría también interpretarse muy formalmente. Desde luego, todavía no resulta claro si todos los elementos tienen la misma importancia en esta estructura, en esta totalidad. No se sabe aún cómo llegamos hasta la estructura. Se puede aceptar la idea que ya en nuestro cerebro todo es interdependiente, que las células nerviosas representan una estructura. Pero nosotros no sabemos bajo qué condiciones funcionan mejor. No es suficiente decir que todo es estructura en nuestro cerebro, sino que hay también que explicar cómo podemos provocar un mejor funcionamiento de estas estructuras. Entonces, es nuestra intención introducir también en esta discusión sobre la estructura la noción de funcionamiento y subrayar una vez más que la estructura se realiza a través del funcionamiento. Es aquí que nos apartamos del pensamiento de Jean Piaget, que considera el funcionamiento como la consecuencia de las estructuras o de las subestructuras mentales pre-establecidas.

Formación de las estructuras perceptivo-psicolingüísticas y sus transformaciones.

¿Cómo se forman las estructuras perceptivas y psicolingüísticas?

¿Son superadas estas estructuras por las distintas transformaciones?

Las estructuras perceptivas y psicolingüísticas se forman:

1) sobre la base de factores óptimos que toman una forma discontinua y

2) las estructuras son superadas en el curso de su realización por distintas transformaciones.

ad 1) El funcionamiento sobre la base de lo óptimo con formas discontinuas.

Las estructuras psicolinguísticas se forman sobre la base de las posibilidades y disposiciones hereditarias del cerebro humano para responder a los estímulos lógicolingüísticos mediante los procedimientos de "filtraje" de los estímulos exteriores. Este "filtraje" es necesario, puesto que la respuesta total (cuantitativamente) al estímulo físico volvería al cerebro humano anárquico, desorganizado y sobrecargado.

Es el sistema cerebral, debido a la inhibición, que permite al cerebro organizarse, respondiendo prioritariamente a los elementos de la estimulación que le son óptimos y que se organizan mejor en sus experiencias anteriores. La respuesta cerebral corresponde entonces más bien a los procesos de eliminación y de estructuración, que a los procesos de asimilación cuantitativa. 
Hemos visto ya que todos los elementos no son igualmente importantes. Los estímulos físicos no llegan en su integridad a la corteza cerebral; las vías neurológicas que van de la corteza hacia la periferia, como la neurofisiología moderna nos enseña, tienen una función inhibitoria. Esto quiere decir que el estímulo físico primero se transforma en vías nerviosas y subiendo siempre hacia la corteza cerebral, pasa por diversas fases de purificación y eliminación. La teoría Gestalt ha subrayado ya hace tiempo este fenómeno cerebral. Para percibir un objeto no es necesario ver todos sus elementos, es suficiente ver la forma. El caso más citado es el del triángulo. Es suficiente ver tres puntos dispuestos como los vértices de un triángulo para percibir todo el conjunto. El fenómeno de discontinuidad se encuentra, pues, ya presente en la Teoría de la Forma. Aunque no tuviéramos delante de nosotros el objeto total, percibiríamos el triangulo en su totalidad porque lo percibiríamos a través de sus elementos óptimos. Esto quiere decir que si todos los elementos se presentan a nuestros sentidos, nuestra corteza reacciona con una elección y no con la suma algebráica de todos sus elementos.

Cuanto más se desarrolla el cerebro humano, tanto mejor actúa mediante los optimales, la eliminación y la estructuración. El bebé que aún no tiene siquiera un mes, comienza ya a "dirigir" los reflejos de su boca hacia el seno de la madre (Piaget). La inhibición, la eliminación y la selección actúan ya. En el transcurso del segundo mes, el niño no chupa más "por casualidad" su mano, sino dirige su boca al dedo pulgar. Es así cómo el cerebro humano se organiza, se estructura y progresa.

Para superar y enriquecer las estructuras perceptivas y psicolingüísticas, es necesario estimular el cerebro con los elementos óptimos para el cerebro, dependiendo esto del nivel de sus adquisiciones. En la teoría verbotonal llamamos esto "optimales". Podemos citar algunos optimales (elementos óptimos) para la adquisición del habla del niño: su capacidad humana para el habla; su alegría por emitir los sonidos y después su alegría y la posibilidad de servirse del habla; su desarrollo motor; su desarrollo táctil, visual y auditivo; el desarrollo de su propiocepción; su desarrollo afectivo e intelectual; su ambiente social y la calidad de este ambiente social.

ad 2) Graduación de los optimales y superación de las estructuras perceptivo-lingüísticas.

Para hacer percibir bien los fonemas al alumno, nos serviremos en las primeras fases, antes que nada, del ritmo y de la entonación. Por ejemplo, si el alumno no puede pronunciar la vocal (y), que es una vocal aguda, le damos esta (y) en una palabra que se encuentra en el vértice de una entonación ascendiente, por ejemplo, "Viens-tu?". Si se trata de una (e) abierta, emplearemos la entonación descendiente. Cuando él ha pronunciado correctamente los fonemas difíciles en las condiciones óptimas (aquéllas que favorecen un buen funcionamiento del cerebro) comienza ya a ser capaz de pronunciar estos mismos fonemas en unas estructuras más complejas y es entonces cuando la primera estructura perceptiva habrá sido superada. La segunda fase, la segunda 
estructura, será así más perfecta, superior a la primera. De este modo el alumno podrá pronunciar los fonemas con cualquier entonación; estas entonaciones le servirán después para corregir una expresividad personal, una comunicación personal.

Lo mismo sucede en la corrección fonética, donde, en principio, ponemos un fonema difícil próximo a los sonidos óptimos. Por ejemplo, si en lugar de pronunciar (y) pronuncia (i), logra corregirse cuando se asocia esta (y) a las consonantes bilabiales. Después podemos poner el fonema cerca de cualquier otro fonema y el alumno pronunciará bien (y). La estructura será esta vez superior y más avanzada que la primera, donde estábamos obligados a utilizar una proximidad limitada y determinada para llegar a una buena o mejor pronunciación.

Cuando usamos aparatos (SUVAG LINGUA) es el mismo fenómeno el que se produce.

En efecto, la octava óptima debe limitar nuestra escucha a un cierto número de frecuencias para evitar demasiada selección de fonemas, lo que conduciría al alumno hacia su lengua materna.

Nos aproximamos al alumno cuando utilizamos la banda óptima correctiva. Esto significa que no nos contentamos tan sólo con la banda óptima, desde el punto de vista de la lengua extranjera, sino que presentamos el fonema en cuestión mediante una banda que es la más lejana de la banda óptima del error cometido por el alumno. Esta banda se llama banda correctiva y en esta fase es la más eficaz para la escucha del alumno. En una fase posterior nos acercamos a la escucha ordinaria (normal), transmitiendo progresivamente el espectro total del habla. Esta ampliación hacia el espectro total y las frecuencias de la zona de conversación es entonces algo contrario a lo de las fases precedentes.

¿Qué es lo que sucede en la superación de las estructuras en el campo del aprendizaje de lenguas extranjeras? ¿Se trata de nuevas organizaciones o reorganizaciones de actividades cerebrales? Antes de responder a esta pregunta pertinente (y también inquietante), vamos a presentar la superación de las estructuras perceptivas en el campo de la rehabilitación auditiva (en las deficiencias graves del oído).

Sabemos que un sordo completo no oye. Pero se puede iniciar la rehabilitación de la comprensión del habla a través del sentido vibro-táctil, sirviéndonos de un aparato (SUVAG) y de un vibrador. En esta primera fase es el único medio de que dispone el sordo completo para poder percibir el habla de forma limitada. Seis meses o un año más tarde, este mismo sordo, que al principio no podía percibir nada a través del oído, comienza a percibir mejor si se combina el vibrador puesto en la mano con los auriculares en la orejas. En la tercera fase puede percibir mejor a través del oído. Ya que al principio se comenzó la rehabilitación auditiva por medio del sentido vibro-táctil (única vía eficaz para el sujeto en cuestión en la primera fase), esto nos ha permitido pasar gradualmente a la segunda y después a la tercera fase. Las estructuras se han visto enriquecidas tanto desde el punto de vista perceptivo como del psicolinguístico. 
Las investigaciones neurofisiológicas por medio del potencial cerebral evocado, han podido demostrar que los deficientes auditivos completos que han conseguido mediante la rehabilitación verbotonal comprender el habla a través del cuerpo (el vibrador puesto en la mano, unido con el aparato SUVAG) habían reorganizado funcionalmente su sistema somato-sensorio. En efecto, las estimulaciones vibrotáctiles alcanzan la latencia de los estímulos auditivos (50 m. seg.), mientras que los oyentes normales, por el mismo tipo de estimulación vibrotáctil (mediante el vibrador en la mano), alcanzaban la latencia más larga y típica para los estímulos táctiles (80-90 m. seg.).

Un estudio neurofisiológico, esta vez mediante el sueño, ha demostrado igualmente que una mejor percepción auditiva y la superación de las estructuras psicolingüísticas dejan señales, al menos funcionales, en el cerebro. El porcentaje del sueño REM y el número de movimientos rápidos de los ojos en el curso del sueño (REM s) están en correlación con la experiencia - maduración.

Las investigaciones en este caso han podido demostrar que los sujetos que suenan por debajo de la media presentan ciertas insuficiencias mentales o de otro tipo. La investigación se ha realizado con dos categorías de niños sordos, de las cuales una (los no rehabilitados con el método verbotonal), comunicaba poco por el habla y no la podía comprender sin lectura labial; y la otra (los rehabilitados con el método verbotonal), hablaba y comprendía el habla sin lectura labial. El porcentaje del sueño en su totalidad para el primer grupo (no rehabilitados con el método verbotonal) alcanzaba el $4 \%-12 \%$. El porcentaje del sueño en el segundo grupo (rehabilitados con el método verbotonal), alcanzó el $22 \%$ y hasta el $24 \%$ de la totalidad. Se observa que la mejor percepción del habla y el habla misma se aproximan en los deficientes auditivos bien rehabilitados al habla de los oyentes, mientras que los deficientes de audición que no comunican por el habla y no la comprenden (sin mirar), quedan en los niveles bajos del porcentaje del sueño en la totalidad.

Era útil, creemos, citar los dos estudios neurofisiológicos que conciernen a los sordos, porque sabemos según diversos estudios que los sujetos que aprenden lenguas extranjeras se comportan como duros de oído. Se podría pues plantear la hipótesis que las estructuras óptimas para la percepción auditiva y la superación de las estructuras perceptivo-psicolinguísticas están ligadas a la organización y la reorganización funcional de nuestra actividad cerebral.

Tales hipótesis son posibles porque en la estructura verbotonal (y por lo tanto también en la metodología SGAV), vemos ante todo el funcionamiento. Ahora bien, el funcionamiento puede partir hacia nuevas organizaciones.

Podemos concluir que el funcionamiento cerebral de las estimulaciones óptimas (y en general de los optimales), conduce a la superación de las estructuras perceptivas y psicolingüísticas. 


\section{Povzetek \\ STRUKTURA V VERBOTONALNI TEORIJI TER PREMAGOVANJE PERCEPTIVNIH IN JEZI- KOVNIH STRUKTUR OB REHABILITACIJI}

Zakonitosti strukture imajo pomembno vlogo tudi v delovanju jezika, se pravi, pri konkretnem izvajanju komunikacijskega dejanja. Treba bi bilo pojasniti, kaj so "strukture delovanja" in kaj je tisto, kar imenujemo "strukturalno razumevanje jezika". Pri tem je treba poudariti, da pojem "struktura" ni istoveten pojmu v znanih strukturalnih teorijah; treba ga je razumeti v običajnem psihološkem smislu. Naše dojemanje se strukturira predvsem na podlagi optimalnih elementov emisije. Ritem in intonacija spadata mednje. Verbotonalna teorija izhaja iz govora. Dojemanje govora teče globalno. Strukturo jezika karakterizira iskanje optimalnega: vsi elementi komunikacije se strukturirajo tako, da bi ta potekala nemoteno in čimbolj učinkovito. To dejstvo nas pripelje do drugačne definicije strukture. Ni pa docela jasno, kako pride do te strukture; domnevamo, da je v naših možganih medsebojna odvisnost in da živčne celice tvorijo neko strukturo. Ne vemo pa, pod kakšnimi pogoji te strukture najbolje funkcionirajo. Ni dovolj, da povemo, da je v naših možganih vse struktura; treba bi bilo pojasniti, kako lahko spodbudimo te strukture $k$ boljšemu delovanju. Avtor želi govoriti predvsem o pojmu delovanja in pri tem poudarja, da nastane struktura $v$ delovanju. S pomočjo raziskovanj na področju rehabilitacije sluha poskuša pokazati, kako se formirajo perceptivno-psiholingvistične strukture in kakšen je način njihovega premagovanja. 